\title{
The theory of endobiogeny: biological modeling using downstream physiologic output as inference of upstream global system regulation
}

\author{
Kamyar M. Hedayat \\ Systems Biology Research Group, 6702 N. Sheridan Road 2N, Chicago, Illinois, USA \\ ${ }^{1}$ Corresponding author \\ E-mail: acquamarine@mac.com \\ Received 1 October 2019; received in revised form 4 June 2020; accepted 16 June 2020 \\ DOI https://doi.org/10.21595/chs.2020.21072
}

Copyright (C) 2020 Kamyar M. Hedayat. This is an open access article distributed under the Creative Commons Attribution License, which permits unrestricted use, distribution, and reproduction in any medium, provided the original work is properly cited.

\begin{abstract}
Living organisms are physical, chemical and biological systems organized in fractal hierarchies of structure and function. Various levels of organization may be the object of study: cell, tissue, organ or whole system. The theory of endobiogeny is a global systems approach to living systems in which the patient as a whole is the object of study. It is a global living systems theory of terrain. The essential activity of the terrain is metabolism: the continuous transformation of energy and matter. According to this theory, the neuroendocrine system is the manager of metabolism and hence the terrain. Modeling the neuroendocrine elements of regulation of the terrain is a key tool in clinical assessment of patients. There are various limits to a strictly quantitative measurement of hormone levels and metabolic activity. Therefore, a modeling system has been developed which uses downstream biomarker output from tissues to assess upstream elements of management of the terrain. This method is called the Biology of Functions. Direct and indirect ratios of biomarkers and indexes have been developed to use quantized data to generate a qualitative analysis of relative capability of the organism. The construction of two direct indexes (genital ratio and genito-thyroid), and one indirect index (evoked histamine) are discussed.
\end{abstract}

Keywords: complex systems theory, cortisol, endobiogeny, endocrine system, genital ratio, genito-thyroid index, neutrophil-to-lymphocyte ratio.

"The problem biologists would like to tackle is that of the integration of physiological functions in living organisms. But as we have seen, in spite of the powerful methods of calculation now available, the quantitative, analytical approach has failed to produce satisfactory results. An interesting possibility seems to lie in the use of a qualitative approach in the identification of the general principles underlying biological systems. These principles may be expected to reveal a functional order that could be further investigated quantitatively." - Gilbert Chauvet, MD, Ph. D. [1]. The Mathematical Nature of the Living World, Chapter 4 - Structural and functional organizations of living organisms: a new look at the structure-functional relationship in biology, p. 124.

\section{Introduction}

The notion of complex systems is ancient. However, complexity, interrelatedness and coherence were largely expressed in philosophical terms [2]. For example, stoic philosopher, Emperor Marcus Aurelius (d.180) observed that animals had "an increasing tendency to unity which does not exist in plants or stones" [3]. Among ancient scientists and physicians, observations of complexity were limited to qualitative and empirical statements. Physician-philosopher Sextus Empiricus (d.210 CE) observed that "unified bodies are such as are controlled by a single hexis (an integral and integrating force of regulation)...(so that) when the finger is cut, the whole body shares in its condition" [4]. Four developments allowed for quantitative evaluation of complex living systems. The first is experimental physiology. From the pioneering work of Bernard, [5] Pavlov and Anokin [6], Selye [7, 8] and others, the dynamic 
nature of biology could be observed and verified. The second is biophysics, which allowed for the principles of physical systems to be applied to biological ones, such as fluid dynamics, hear rate variability and neuronal electrical output [9-11]. The third is macro-biochemistry: in vivo output sampling of biological fluids, e.g. prostate specific antigen and urinary electrolytes [12]. This allows for quantization of gross metabolic activity through measurement of biomarkers. The fourth is micro-biochemistry, e.g. high throughput metabolomics [13]. This allows for massive data acquisition regarding thousands of complex metabolic actions within a single cell.

Complex biological organisms are composed of nested subsystems, both structurally and functionally. Each subsystem is a system unto itself, with intrinsic function, be it a cell, tissue or organ. Some systems are discrete and anatomical, such as the liver. Their coherence is proscribed by space and geometry. Others systems are non-discrete and functional, free of space and specific architecture, such as the immune system [1]. All systems in complex organisms are related through hierarchical levels of organization which integrate and interrelate the systems across space and time. The global living system overrides the intrinsic activity of the subsystems in order to preserve the material (structural) and functional integrity of the whole. In clinical medicine, the patient is the global system, not the cell, or cardiovascular system or any other particular subsystem. Therefore, the whole patient as a global system needs to be the object of ultimate study and the end point of integration of data.

The great challenge in applying systems biology to clinical medicine are three. The first is a theory of terrain that integrates functioning of the sub-systems into a coherent concept of the whole. The human organism is more than the individual metabolic activity of trillions of cells. The second is integration of the various quantitative assessments of biological activity in order to generate actionable data at the bedside. The difficulty in this case is that biochemical, biophysical, and metabolic assessments are measured in divergent ways: voltage, osmolality, concentration gradient, compliance, resistance, etc. Neither assemblage nor amalgamation of this data per se leads to an understanding of the whole in its expressed dynamical function. The third is a qualitative interpretation of quantitative data. The functioning of complex living systems is the sum of a series of non-deterministic binary possibilities, viz. catabolism and anabolism, or, growth and anti-growth, which are expressed simultaneously in various proportions. Therefore, quantitative data should be evaluated in a qualitative fashion. The true functioning of the organism, and its subsystems, is determined by the level of nested or interrelated activities relative to that of their counter-activities, expressed as ratios. In sum, what is needed in clinical practice is an approach to systems biology based on the whole person, which is quantitative in measurement but qualitative in analysis.

\section{Theory of endobiogeny: global theory of terrain}

The theory of endobiogeny is a global systems theory of terrain [14]. The founder of the theory of endobiogeny, Dr. Christian Duraffourd defined terrain as the "functional expression of the structural constitution in its internal equilibrium in the face of endogenous and exogenous aggressions" [15]. Structure is the phenotypic expression of genetic potential in its current, dynamic materialization. Function is the dynamic expression of genetically determined possibility in a non-deterministic field of probability. The terrain is maintained through the process of metabolism. Metabolism is the sum of all actions related to the transformation of energy and matter in biological systems. It ensures both the material integrity and functional dynamism of the terrain. Every unit of function has intrinsic metabolic requirements. The global organism is a series of nested, hierarchical arrangement of units. Something manages the coherency of metabolic activity across all units of function based on three qualities: 1) constancy of action, 2) ubiquity of action and 3) auto-regulation. The endocrine system is the only system to meet these criteria. Therefore, the endocrine system is the manager of the terrain [15].

The autonomic nervous system calibrates and sequences timing, duration, amplitude and intensity of endocrine function. Thus, in practical terms, we refer to the "neuroendocrine" system 
as the manager of metabolism and hence terrain [15]. The neuroendocrine system, therefore, is the primary focus of evaluation in Endobiogeny. The central nervous system (CNS) is also important, but is not the manager of the terrain. It is a surveyor and assessor of internal and external states and conditions in their permanent flux [15]. It creates existential hierarchies of values that assist the neuroendocrine system in its management of metabolism and terrain. The CNS itself is influenced by engrammatic mental perceptions and emotional reactions [16]. In summary, there are three types of upstream influences on metabolism: the neuroendocrine system, the CNS, and, perception and reaction. Metabolism occurs in a "black box" of cellular function, impossible to monitor in real time in the living human. What may be assessed is the downstream output of biomarkers derived from the metabolic activity. Endobiogeny utilizes the third type of quantitative assessment-macro-biochemistry-to infer the sum of major upstream influencers on metabolism.

There are two reasons why the upstream regulators are not directly measured. The first is that it is technically impossible to measure all factors simultaneously in real time. The second is that quantitative measurement of circulating hormones is not a valid reflection of the regulation of metabolism, which we have discussed in detail elsewhere [14]. Briefly, we cite some major issues. The sum of all agonistic and antagonistic hormones determine net effects of metabolism. Assessment of serum levels of individual hormones arrayed side by side in analysis will not determine their net effect on tissue-level metabolism. That is because individual variations in receptor subtype and polymorphisms exist, such that the actions of hormones cannot be linearly associated with their quantitative serum concentration [17-19]. Finally, anticipated effects of hormones can be impaired by persistent organic pollutants [20] or disrupted by xenohormones [21].

\section{Biology of functions: application of downstream output}

The biology of functions (BoF) is a modeling system derived from the theory of endobiogeny. The BoF calculates the qualitative function of the human organism in its dynamic state of phenotypic expression. It uses downstream serum biomarkers obtained by venous blood draw while the patient is in a fasting state $[15,22]$. A number of biomarkers clearly linked to upstream influences on metabolism are used for input. The essential ones are: red blood cells (RBC), white blood cells (WBC), percent differential of WBC (neutrophils, lymphocytes, monocytes, eosinophils and basophils), platelets, hemoglobin, potassium, calcium, sodium, chloride, total protein, thyroid stimulating hormone (TSH), lactate dehydrogenase (LDH), creatine kinase total (CK), osteocalcin, and alkaline phosphatase bone isoenzyme (APBi). Of these, LDH, CK, osteocalcin and APBi are normalized to an internal standard based on the high and low of the normal ranges reported by the laboratory. When the TSH is $<0.5$, then 0.5 must be used as an arbitrary value to prevent extremely large deviations during the calculation of indexes. When TSH is $>4.5$, an internal transformation of TSH is performed to separate out the vertical feed forward activity of TSH on the thyroid from other activities performed by the thyrotropic system.

Once serum biomarkers have been resulted, they are combined by ratios to form direct indexes. Direct indexes may be combined with other indexes and/or additional biomarkers to form indirect, or, meta indexes (c.f. evoked histamine index, discussed later in the article). The normal values for indexes are initially derived through simple mathematical ratios of the normal values of the biomarkers according to the values for the adult female of child-bearing age. This is because all fetuses are by default programmed to become female if not for the activation of sex-determining region of $Y$ chromosome and Müllerian inhibiting factor [19]. Modifications in values are made for adult males and children according to certain programmatic differences in the levels of activity of certain elements of physiology, such as cortisol, estrogens, growth hormone, histamine, etc. through various phases of life.

The initial index value represents the general, current capabilities of the organism during adaptive metabolism, referred to as the function value. Through an internal transformation process, a structure value is derived. This represents the capabilities of the organism in basal metabolism. 
The distinction serves a useful purpose for the clinician using the system. Some disorders are disfunctions of basal metabolism, such as certain adenocarcinomas and menstrual disorders. Others are disorders of adaptive metabolism, such as asthma and certain autoimmune disorders.

There are several advantages to using downstream biomarkers as inference of upstream regulation of the terrain. First, quantifiable biomarkers allow for objective, repeatable evaluations of the terrain across time. Second, it allows for qualitative, polyfactorial assessment. Third, it allows for personalization of treatment by evaluating the specific element(s) within the construct of the meta-formulas most implicated for disequilibrium. For example, asthmatics may have insufficiency of cortisol, hyperfunctioning immune activity, hyperfunctioning autonomic nervous system activity, or some combination of all three. The indexes of cortisol, histamine and interleukins, respectively, allow for assessment of the equilibrium state of each factor. Based on the absolute or relative derangement of values, appropriate treatments can be selected. This is a novel approach that personalizes to phenotype rather than symptoms or genotype. In sum, the use of downstream biomarkers brings the clinical evaluation closer to the unity of the global expression of life that is the living patient.

There are disadvantages and limitations to the biology of functions worth noting. First, assumptions are made about how strongly associated specific biomarkers are to specific upstream regulators (c.f. genital ratio). Second, output from a single tissue (e.g. bone marrow - c.f. genital ratio) is assumed to be representative of the effects of hormones on all tissues. Third, the endobiogenic method is limited to general trends of the global terrain, not specific organs or tissues. Finally, the BoF does not evaluate real-time time scales of physiologic function. It represents a sum of activity over time - the specific cycle of time depends on the physiologic activity being modeled. With respect to acute adaptation, the results can change hourly. With respect to longer processes, such as chronic stress or pre-cancerous dysplasia, the indexes may change over weeks or months when blood samples are repeated.

The BoF is ultimately a tool submitted to the process of clinical medicine. According to the theory of endobiogeny, each disorder arises from a polyfactorial series of imbalances, particular to the system or tissue. It is through the triangulation of history, examination and biology of functions that individual observations are assembled into a clinically relevant conclusion. For example, consider a patient with a cutaneous allergic response. On physical exam, the histaminic response may be replicated, or other signs determined of local vs. systemic expression of histamine. On the BoF, the terrain is evaluated not only for an elevated histamine index, but also tissue-level cortisol activity, immune function, etc. If the histamine index is elevated, then the local allergic response derives from global system imbalances. If the index is normal or low, then the local allergic response is derived purely from local responses to allergens.

\section{Construction of direct indexes}

Androgens and estrogens are anabolic hormones that regulate cell metabolism. They stimulate DNA transcription elements related to protein-rich structures and activities, such as enzymes, as well as regulation of energy metabolism [23, 24]. Because of the limitation of quantitative levels of hormones and their effects on metabolism, a direct index was derived to approximate the global effects of these anabolic steroids. It has been observed that androgens stimulate the production of RBC's in the bone marrow, [25] as estrogens do for WBC's [26]. The Genital Ratio (GR) is defined as the relative activity of androgens on tissue metabolism during adaptation in relationship to that of estrogens:

Genital Ratio $=$ Red blood cells $\times 103$ per $\mu \mathrm{L} /$ White blood cells per $\mu \mathrm{L}$.

The normal value is $0.8-0.95$ for men, $0.7-0.85$ for women. It considers the actions of androgens and estrogens on bone marrow to be indicative of the general activity of these anabolic steroids throughout the body [15]. 
Neutrophils and lymphocytes are white blood cells involved in immunity and inflammation. The Neutrophil-to-lymphocyte ratio is a long-studied ratio in medicine. Researchers have observed that a higher value of the ratio is related to systemic inflammation and mortality in various disorders [27, 28]. Independent from this line of research, Christian Duraffourd, founder of the theory of Endobiogeny, [15] developed the genito-thyroid index (GTi):

Genito-thyroid index $=\%$ Neutrophils / \% Lymphocytes.

The normal value is 1.5-2.5 in men and women. It is defined as the relative activity of estrogens in relationship to that of the thyroid. Estrogens proliferate neutrophils and TSH proliferates T-lymphocytes in the thymus [29, 30]. The higher the index, the greater the tendency towards autoimmune disease and/or systemic inflammation in response to an aggression [31, 32]. The lower the index, the greater the tendency towards atopic disease and localized inflammation in response to an aggression [33]. We have recently described the relationship of these biomarkers to the endocrine system, and demonstrated its relationship to chronic heart failure and cancer survivors $[34,35]$.

The peripheral thyroid hormones, thyroxine (T4) and tri-iodothyronine (T3) are key for oxidative metabolism for ATP production.[36] Coregulators can amplify or diminish their effects.[37] Thus, there is not a linear association between free quantitative serum concentration and action on metabolism. The Krebs cycle requires pyruvate for de novo ATP production. The enzyme lactate dehydrogenase (LDH) is key in the glycolytic pathway for ATP production. The enzyme creatine kinase (CK) phosphorylates ADP to ATP when there is insufficient time for de novo ATP production [38]. The more efficient the action of peripheral thyroid hormones is, the greater the ratio of LDH to CPK will be. The less effective thyroid activity is, the lower the ratio will be (Table 1) [39]. The thyroid index is defined as the effective metabolic activity of peripheral thyroid hormones at the cell level:

Thyroid index $=$ LDH IU per liter $/$ CK units per Liter.

The normal range is 3.5-5.5 in men and women, after LDH and CPK values are have been normalized for internal calculation (c.f. Biology of Functions: application of downstream output).

Table 1. Lactate dehydrogenase and Creatine Kinase activity in patients with various states of thyroid function

\begin{tabular}{|c|c|c|c|}
\hline Condition & LDH & $\mathrm{CK}$ & Ratio \\
\hline Hyperthyroidism & 233.80 & 88.37 & 2.65 \\
\hline Subclinical Hyperthyroidism & 227.81 & 105.98 & 2.15 \\
\hline Normal controls & 202.85 & 102.19 & 1.99 \\
\hline Subclinical hypothyroidism & 340.38 & 179.80 & 1.89 \\
\hline Hypothyroidism & 421.00 & 389.90 & 1.08 \\
\hline \multicolumn{4}{|c|}{$\begin{array}{l}\text { Modified from McGrowder D. A., et al., Nig. J. Clin. Pract., 14(4), } 2011 . \\
\text { CK: Creatine kinase, LDH: Lactate dehydrogenase }\end{array}$} \\
\hline
\end{tabular}

\section{Construction of indirect indexes}

As an internally coherent theory of terrain and integrative physiology, the indexes of the biology of functions have two advantages. The first is a qualitative modeling of physiology. The second is an additive, multifactorial process of building complex, meta-indexes from direct indexes. In other words, foundational observations of metabolism and adaptation are built upon to generate indirect indexes that are reflective of increasingly complex and interrelated physiologic processes.

The evoked histamine index is an indirect index composed of both direct biomarkers, direct indexes and indirect indexes. 
Evoked histamine $=$ Eosinophil $\% \times$ Platelets $\times 103$ per $\mu \mathrm{L} \times$ Adaptation index $\times$ Androgenic index / Cortisol index.

It is defined as the total level of circulating histamine (activity). The normal value is 20-60 for women, and 36-76 for men. The adaptation, androgenic and cortisol indexes are discussed elsewhere $[15,22]$. The Evoked histamine index is developed based on a number of well-known physiologic observations. For example, eosinophils [40], androgens [41] and platelets [42, 43] increase histamine activity. Conversely, cortisol diminishes histamine release and inhibits eosinophils [44-53].

\section{Conclusions}

The theory of endobiogeny is a global systems theory of terrain. It evaluates human physiology based on the principles of interrelatedness and integration of various subsystems within the global system. From this theory, a biological modeling system has been derived called the biology of functions. The biology of functions uses quantitative measurements of biomarkers. It considers this data as the downstream metabolic output of tissues and organs in the body based on upstream influencers: the neuroendocrine system and central nervous system. The biomarkers are related qualitatively as ratios to infer the activity of the upstream regulators. The BoF is a novel approach to modeling human physiology. It allows from both a quantitative and qualitative assessment of human physiology, current and future risk of pathophysiology and personalized methods of assessment for maintaining health. Further clinical studies are on-going to assess the robustness of the biology of functions across various physiologic states, conditions and disorders.

\section{References}

[1] Chauvet G. The Mathematical Nature of the Living World: the Power of Integration. World Scientific, New Jersey, 2004.

[2] Sambursky S. Physics of the Stoics. Princeton University Press, Princeton, 1987, p. 153.

[3] Marcus A., Haines C. R. Marcus Aurelius. Harvard University Press, Cambridge, 1930.

[4] Bury R. G. Sextus Empiricus. Harvard University Press, Cambridge, 1933.

[5] Claude B. An Introduction to the Study of Experimental Medicine. Dover Publications, 1949, p. 226.

[6] Red'ko V. G., Prokhorov D. V., Burtsev M. B. Theory of functional systems, adaptive critics and neural networks. Proceedings of International Joint Conference on Neural Networks, 2004.

[7] Selye H. The Significance of the Adrenals for Adaptation. Science, Vol. 85, Issue 2201, 1937, p. 247-8.

[8] Selye H. Stress and the general adaptation syndrome. British Medical Journal, Vol. 1950, Issue 1, 4667, p. 1383-1392.

[9] McCraty R., et al. Synchronization of human autonomic nervous system rhythms with geomagnetic activity in human subjects. International Journal of Environmental Research and Public Health, Vol. 14, Issue 7, 2017, p. 1-18.

[10] von Kanel R., et al. Relationship between heart rate variability, interleukin-6, and soluble tissue factor in healthy subjects. Brain Behav Immun, Vol. 22, Issue 4, 2008, p. 461-468.

[11] Chauvet G. A. On the mathematical integration of the nervous tissue based on the S-propagator formalism: I. Theory. Journal of Integrative Neuroscience, Vol. 1, Issue 1, 2002, p. 31-68.

[12] Rao A. R., Motiwala H. G., Karim O. M. The discovery of prostate-specific antigen. British Journal of Urology Int, Vol. 101, Issue 1, 2008, p. 5-10.

[13] Sridharan G. V., et al. Prediction and quantification of bioactive microbiota metabolites in the mouse gut. Nature Communications, Vol. 5, 2014, p. 5492.

[14] Lapraz J. C., Hedayat K. M. Endobiogeny: a global approach to systems biology (part 1 of 2). Globalb Advances in Health and Medicine, Vol. 2, Issue 1, 2013, p. 64-78.

[15] Hedayat K., Lapraz J. C. The Theory of Endobiogeny: Global Systems Thinking and Biological Modeling for Clinical Medicine. Vol. 1, Academic Press, Philadelphia, 2019.

[16] Newman S. P., Epstein R. Current Perspectives in Dysphasia. Churchill Livingstone, Edinburgh, New York, 1985, p. 237. 
[17] Kadmiel M., Cidlowski J. A. Glucocorticoid receptor signaling in health and disease. Trends in Pharmacological Science, Vol. 34, Issue 9, 2013, p. 518-530.

[18] Simoni M., Casarini L. Mechanisms in endocrinology: Genetics of FSH action: a 2014-and-beyond view. European Journal of Endocrinology, Vol. 170, Issue 3, 2014, p. 91-107.

[19] Nussey S., Whitehead S. Endocrinology: An Integrated Approach. BIOS Scientific Publishers, Oxford, 2001.

[20] Lee D. H., Jacobs D. R. Jr., Association between serum concentrations of persistent organic pollutants and gamma glutamyltransferase: results from the National Health and Examination Survey 1999-2002. Clinical Chemistry, Vol. 52, Issue 9, 2006, p. 1825-1827.

[21] Węsierska-Gadek J. Endocrine disruptor contaminants in water and their adverse effects in humans. Ecohydrology and Hydrobiology, Vol. 6, Issues 1-4, 2006, p. 233-242.

[22] Lapraz J. C., Hedayat K. M., Pauly P. Endobiogeny: a global approach to systems biology (part 2 of 2). Global Advances in Health and Medicine, Vol. 2, Issue 2, 2013, p. 32-44.

[23] Cui J., Shen Y., Li R. Estrogen synthesis and signaling pathways during aging: from periphery to brain. Trends in Molecular Medicine, Vol. 19, Issue 3, 2013, p. 197-209.

[24] Bennett N. C., et al. Molecular cell biology of androgen receptor signalling. International Journal of Biochemistry and Cell Biology, Vol. 42, Issue 6, 2010, p. 813-827.

[25] Naets J. P., Wittek M. The mechanism of action of androgens on erythropoiesis. American Journal of Physiology, Vol. 210, Issue 2, 1966, p. 315-320.

[26] Cruickshank J. M., et al. The relationship of total and differential leukocyte counts with urinary oestrogen and plasma cortisol levels. British Journal of Obstetrics and Gynaecology, Vol. 77, Issue 7, 1970, p. 634-639.

[27] An X., et al. Elevated neutrophil to lymphocyte ratio predicts survival in advanced pancreatic cancer. Biomarkers, Vol. 15, Issue 6, 2010, p. 516-522.

[28] Tomita M., et al. Elevated preoperative inflammatory markers based on neutrophil-to-lymphocyte ratio and C-reactive protein predict poor survival in resected non-small cell lung cancer. Anticancer Research, Vol. 32, Issue 8, 2012, p. 3535-3538.

[29] von Dadelszen P., et al. Maternal neutrophil apoptosis in normal pregnancy, preeclampsia, and normotensive intrauterine growth restriction. American Journal of Obstetrics and Gynecology, Vol. 181, Issue 2, 1999, p. 408-414.

[30] Sekulic M., et al. Immunoreactive TSH cells in juvenile and peripubertal rats after estradiol and human chorionic gonadotropin treatment. Acta Histochemica, Vol. 108, Issue 2, 2006, p. 117-123.

[31] Hussein O. A., El Toukhy M.-A., El Rahman H.-S. Neutrophil CD64 expression in inflammatory autoimmune diseases: its value in distinguishing infection from disease flare. Immunology Investigations, Vol. 39, Issue 7, 2010, p. 699-712.

[32] Zahorec R. Ratio of neutrophil to lymphocyte counts-rapid and simple parameter of systemic inflammation and stress in critically ill. Bratisl Lek Listy, Vol. 102, Issue 1, 2001, p. 5-14.

[33] Gelfand E. W., et al. Spectrum of T-lymphocyte activities regulating allergic lung inflammation. Immunology Review, Vol. 278, Issue 1, 2017, p. 63-86.

[34] Hedayat K., et al. Genito-Thyroid index: a global systems approach to the neutrophil-to-lymphocyte ratio according to the theory of Endobigoeny applied to ambulatory patients with chronic heart failure. Journal of Cardiology and Clinical Research, Vol. 5, Issue 1, 2017, p. 1091-1097.

[35] Buehning L. J., et al. A novel use of biomarkers in the modeling of cancer activity based on the theory of endobiogeny. Global Advances in Health and Medicine, Vol. 3, Issue 4, 2014, p. 55-60.

[36] Dorsa K. K., Santos M. V., Silva M. R. Enhancing T3 and cAMP responsive gene participation in the thermogenic regulation of fuel oxidation pathways. Arquivos Brasileiros de Endocrinologia e Metabologia, Vol. 54, Issue 4, 2010, p. 381-389.

[37] Mendoza A., Hollenberg A. N. New insights into thyroid hormone action. Pharmacologic Therapy, Vol. 173, 2017, p. 135-145.

[38] Mcleish M. J., Kenyon G. L. Relating structure to mechanism in creatine kinase. Crit Review of Biochemistiry and Molecular Biology, Vol. 40, Issue 1, 2005, p. 1-20.

[39] Mcgrowder D. A., et al. Serum creatine kinase and lactate dehydrogenase activities in patients with thyroid disorders. Nigerian Journal of Clinical Practice, Vol. 14, Issue 4, 2011, p. 454-459.

[40] Jutel M., et al. Immune regulation by histamine. Current Opinions in Immunology, Vol. 14, Issue 6, 2002, p. 735-740. 
[41] Chen W., et al. Human mast cells express androgen receptors but treatment with testosterone exerts no influence on IgE-independent mast cell degranulation elicited by neuromuscular blocking agents. Experimental Dermatology, Vol. 19, Issue 3, 2010, p. 302-304.

[42] Graham H. T., et al. Distribution of histamine among leukocytes and platelets. Blood, Vol. 10, Issue 5, 1955, p. 467-481.

[43] Totani L., Evangelista V. Platelet-leukocyte interactions in cardiovascular disease and beyond. Arteriosclerosis, Thrombosis and Vascular Biology, Vol. 30, Issue 12, 2010, p. 2357-2361.

[44] Ohtsu H. Progress in allergy signal research on mast cells: the role of histamine in immunological and cardiovascular disease and the transporting system of histamine in the cell. Journal of Pharmacologic Science, Vol. 106, Issue 3, 2008, p. 347-353.

[45] Basu Mallik K. C., Bhattacharya D. K. The role of histamine in tropical eosinophilia. Journal of the Indian Medical Association, Vol. 33, 1959, p. 260-263.

[46] Bharadwaj T. P., Gaitonde B. B., Jhala H. I. Histamine and tropical eosinophilia. Indian Journal of Medical Research, Vol. 47, 1959, p. 377-381.

[47] el Hawey A.-M., Selim A. S., Mousa A. H. Plasma histamine level and its relation to blood eosinophilia in bilharzialcases. Journal of the Egyptian Medical Association, Vol. 53, Issue 7, 1970, p. 530-537.

[48] Ganatra R. D., Gaitonde B. B., Sheth U. K. Blood levels of histamine in relation to eosinophilia. Indian Journal of Medical Science, Vol. 14, 1960, p. 328-334.

[49] Hungerford G. F. Role of Histamine in Producing the Eosinophilia of Magnesium Deficiency. Experimental Biology and Medicine, Vol. 115, 1964, p. 182-185.

[50] Josefsson B. Studies on eosinophil granulocytes. V. Evidence against the role of histamine as a mediator of eosinophilia in the uterus of the rat. Acta Endocrinology (Copenhagen), Vol. 58, Issue 3, 1968 , p. 532-536.

[51] Litt M. Studies in experimental eosinophilia. X. Dissociation of histamine-induced vascular eosinophilia and antigen-induced tissue eosinophilia. Journal of Reticuloendothelial Society, Vol. 14, Issue 2, 1973, p. 158-170.

[52] Litt M. Studies in experimental eosinophilia. XI. Dependence of eosinophilia, apparently induced by histamine, on acidity. International Archives of Allergy and Applied Immunology, Vol. 50, Issue 4, 1976, p. 473-487.

[53] Smith M. J., Garrett R. H. A heretofore undisclosed crux of eosinophilia-myalgia syndrome: compromised histamine degradation. Inflammation Research, Vol. 54, Issue 11, 2005, p. 435-450.

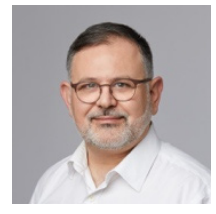

Kamyar Hedayat received his medical doctorate at Wayne State University, Detroit, USA in 1999, post-graduate training in pediatrics at William Beaumont Hospital in 2001 and fellowship training in pediatric intensive care medicine from Stanford University in 2004. In addition to his private medical practice, he is chief scientific officer and co-founder of Systems Biology Research group. His current research interests include global systems integrative physiology and its application to hospital medicine including myocardial infarction and SARS CoV-2. 